\title{
COMPLETION AND SAMUEL COMPACTIFICATION OF NEARNESS AND UNIFORM FRAMES
}

\author{
BERNHARD BANASCHEWSKI AND ALEŠ PULTR
}

\begin{abstract}
It is shown that the familiar description of the completion of a uniform frame in terms of its Samuel compactification can be extended to arbitrary nearness frames. This is achieved by means of the following new notion, a variant of compactness, for regular frames: such a frame will be called near-compact if it is complete in some totally bounded nearness. This leads to a natural concept of the Samuel near-compactification for arbitrary nearness frames which is then shown to play exactly the same rôle in the general setting which the Samuel compactification plays for uniform frames.
\end{abstract}

Keywords: complete nearness and uniform frames, compact, near-compact, Samuel (near-)compactification

AMS Subject Classification (2000): O6D22, 54E17, 54E15

\section{INTRODUCTION}

There are fundamental differences between the descriptions of the completion of

(1) uniform frames by means of their Samuel compactification ([4]), and

(2) general nearness frames directly as a quotient of their down-set frame ([5], see also [12]).

Most obviously, (1) is a two-stage process, involving, first, a suitable compactification and then a certain modification of that, while (2), which presents the completion in terms of certain downsets of the underlying frame, produces the result in one step.

Note. In fact, (1) can be viewed as a three-stage process: the compactification consists of, first, taking the ideal frame, which is a quotient of the down-set frame and next the subframe of strongly regular ideals; then one modifies that as a quotient again. Thus the procedure follows the pattern

Thanks go to the projects MSM 0021620838 and 1M0545 of the Ministry of Education of the Czech Republic. 
quotient frame $>$ subframe $>$ quotient frame.

In (2) the whole procedure is just taking a quotient, without any subframe construction involved. But this is only a technical affair, not really important.

A more fundamental and seemingly unsurpassable difference lies in the use of a compactification, specific for uniformities carried by completely regular frames. More general nearnesses are carried by general regular frames for which there is no suitable compactification (for obvious reasons: representing a regular frame $L$ as a quotient of a compact regular frame makes $L$ completely regular).

The following account shows, however, that both the above descriptions can be dealt with as one procedure, the first being a special case. The key to this lies in a natural notion generalizing compactness to suit mere regular frames. Recall the classical characteristics of compact metric spaces as the complete totally bounded ones (which in a sense precedes the cover definition). Compact regular frames are indeed precisely those that admit a complete totally bounded uniformity. If we ask about admitting a complete totally bounded nearness, we obtain a more general concept which we call near-compactness. Now each nearness frame has a canonical near-compactification (we call it the Samuel near-compactification) that can serve as an intermediate step for a completion of type (2), providing a modification quite analogous to the procedure in (1). Moreover, for uniform frames this Samuel near-compactification coincides with the Samuel compactification (represented, by the way, directly as a quotient of the down-set frame, surpassing also the technical difference mentioned in the Note above).

\section{Preliminaries}

1.1. Recall that a frame $L$ is a complete lattice satisfying the distribution law

$$
(\bigvee A) \wedge b=\bigvee\{a \wedge b \mid a \in A\}
$$

for all $A \subseteq L$ and $b \in L$. A frame homomorphism $h: L \rightarrow M$ preserves all finite meets and all joins. The top of $L$ will be denoted by $1_{L}$ or simply by 1 , the bottom will be denoted by $0_{L}$ resp. 0 .

We will use the standard notation concerning partially orderd sets such as $\downarrow a=\{x \mid x \leq a\}$ and $\downarrow M=\{x \mid x \leq m$ for some $m \in M\}$ In particular, a set $M$ is called a downset if $\downarrow M=M$. For any frame $L$ we put $\mathfrak{D} L$ for the frame of all non-void downsets of $L$. For any frame homomorphism, $h_{*}: M \rightarrow L$ will be its right (Galois) adjoint, that is, $h_{*}(a)=\bigvee\{x \in L \mid h(x) \leq a\}$. 
Factorizing a frame $L$ by a congruence leads to a so called "closure system", or the set of saturated elements, $S \subseteq L$ that is, in particular, closed under all meets. Thus we have for each $a \in L$

$$
\nu(a)=\bigwedge\{s \in S \mid a \leq s\} \in S .
$$

The mapping $\nu: L \rightarrow L$ given by this is usually referred to as the nucleus associated with the congruence.

For details and more about frames see, e.g., [11, 14].

1.2. A cover of a frame $L$ is a subset $A \subseteq L$ such that $\bigvee A=1$. If $A, B$ are covers we set

$$
A \wedge B=\{a \wedge b \mid a \in A, b \in B\},
$$

obviously it is a cover as well.

For a cover $A$ of $L$ and an element $b \in L$ set

$$
A b=\bigvee\{a \in A \mid a \wedge b \neq 0\}
$$

and if $B$ is another cover we write

$$
A B=\{A b \mid b \in B\} .
$$

A cover $A$ refines $B$ (notation $A \leq B$ ) if for every $a \in A$ there is a $b \in B$ such that $a \leq b$. One says that $A$ is a star-refinement of $B$ if $A A \leq B$, and writes $A \leq{ }^{*} B$.

If $\mathcal{A}$ is a system of covers we set

$$
a \triangleleft_{\mathcal{A}} b \text { if there is an } A \in \mathcal{A} \text { such that } A a \leq b \text {; }
$$

one speaks of the strong inclusion associated with $\mathcal{A}$.

1.3. A nearness $([8,5]) \mathcal{A}$ on $L$ is a non-void set of covers such that (N1) if $A \in \mathcal{A}$ and $A \leq B$ then $B \in \mathcal{A}$,

(N2) if $A, B \in \mathcal{A}$ then $A \wedge B \in \mathcal{A}$, and

(N3) for each $a \in L, a=\bigvee\left\{b \mid b \triangleleft_{\mathcal{A}} a\right\}$.

If, moreover,

(U) for every $A \in \mathcal{A}$ there is a $B \in \mathcal{A}$ such that $B B \leq A$, one speaks of a uniformity $([9,10,5,14])$. Note that

$$
\text { if } \mathcal{A} \text { is a uniformity then } \triangleleft_{\mathcal{A}} \text { interpolates. }
$$

The pair $(L, \mathcal{A})$ is then referred to as a nearness resp. uniform frame.

A frame homomorphism $f: L \rightarrow M$ is a uniform homomorphism $(L, \mathcal{A}) \rightarrow(M, \mathcal{B})$ if for all $A \in \mathcal{A}, f[A] \in \mathcal{B}$.

We speak of a basis of nearness resp. uniformity $\mathcal{A}$ on $L$ if $\{B \mid B \geq$ $A \in \mathcal{A}\}$ is a nearness resp. uniformity. If the nearnesses on $L$ and $M$ are determined by bases $\mathcal{A}, \mathcal{B}, f$ is uniform iff $\forall A \in \mathcal{A} \exists B \in \mathcal{B}, B \leq f[A]$. 
For more about uniform and nearness frames see, e.g., $[9,5,14]$.

1.4. Recall the notation

$$
a \prec b \quad \text { for } \exists c, a \wedge c=0 \& b \vee c=1,
$$

and

$$
a \prec b
$$

if there exist $a_{r}$ for rational $r$ in the unit interval such that

$$
a=a_{0}, b=a_{1}, \text { and } r<s \Rightarrow a_{r} \prec a_{s} .
$$

A frame $L$ is regular resp. completely regular if

$$
\forall a \in L, \quad a=\bigvee\{b \mid b \prec a\} \quad \text { resp. } \quad a=\bigvee\{b \mid b \prec a\} .
$$

A frame admits a nearness iff it is regular, and in that case in particular

$$
\mathfrak{C} L=\{C \mid C \text { a cover of } L\}
$$

is a nearness on $L$, referred to as the fine nearness on $L$.

A frame admits a uniformity iff it is completely regular. Then it has a largest uniformity

$$
\mathfrak{F} L=\bigcup\{\mathcal{A} \mid \mathcal{A} \text { a uniformiity on } L\}
$$

called the fine uniformity on $L$. Note that $\mathfrak{F} L$ is not necessarily $\mathfrak{C} L$.

Further, it is a well known and simple fact that $a \triangleleft_{\mathcal{A}} b \Rightarrow a \prec b$ and if $\mathcal{A}$ contains all the two-element covers then $\triangleleft_{\mathcal{A}}=\prec$ (making $\triangleleft_{\mathcal{A}}=\prec$ whenever $\triangleleft_{\mathcal{A}}$ interpolates). Moreover, the fine uniformity contains in particular all the $\left\{a^{*}, b\right\}$ with $a \prec b$ where $a^{*}$ is the pseudocomplement of $a$ (see e.g. [14], 12.2.2), yielding $\triangleleft_{\mathfrak{F} L}=\prec$.

1.5. For a nearness $\mathcal{A}$ set

$$
\operatorname{tb} \mathcal{A}=\{A \mid A \geq B \in \mathcal{A}, B \text { finite }\} .
$$

If $A x \leq y$ with $A \in \mathcal{A}$ then $B=\{A x, \bigvee\{a \in \mathcal{A} \mid a \wedge x=0\}\}$ is in tb $\mathcal{A}$ and $B x=A x \leq y$; thus, $\triangleleft_{\mathrm{tb} \mathcal{A}}=\triangleleft_{\mathcal{A}}$ and hence tb $\mathcal{A}$ satisfies (N3) and is a nearness again.

A nearness $\mathcal{A}$ (or the nearness frame $(L, \mathcal{A})$ is said to be totally bounded if $\operatorname{tb} \mathcal{A}=\mathcal{A}$.

By Isbell [10] (the proof in the book - 30 on p.23 - is in classical terms, but it can be obviously modified for the point-free context) (1.5.1) if $\mathcal{A}$ is a uniformity then $\operatorname{tb} \mathcal{A}$ is a uniformity as well. 
The identity carried (uniform) homomorphisms

$$
(L, \operatorname{tb} \mathcal{A}) \rightarrow(L, \mathcal{A})
$$

is the coreflection map from the category of nearness (resp. uniform) frames to that of totally bounded nearness (resp. uniform) frames.

1.6. Samuel compactification. A compact regular frame $L$ admits precisely one nearness, namely $\mathfrak{C} L$, and it is a uniformity, hence $\mathfrak{C} L=\mathfrak{F} L(=\mathrm{tb} \mathfrak{C} L=\operatorname{tb} \mathfrak{F} L)$. Thus, for such $L$ every frame homomorphism $M \rightarrow L$ is a uniform homomorphism for any $(M, \mathcal{A})$. Thus the category of compact regular frames can be viewed as a full subcategory of the category of uniform frames.

An ideal $J$ in a uniform frame $(L, \mathcal{A})$ is said to be $\mathcal{A}$-regular (briefly, regular) if

$$
\forall a \in J, \exists b \in J \quad \text { such that } a \triangleleft_{\mathcal{A}} b .
$$

The set $\mathfrak{R}(L, \mathcal{A})$ of all regular ideals, ordered by inclusion, is a subframe of the ideal frame of $L$ and hence it is compact. The homomorphisms

$$
v_{(L, \mathcal{A})}=(J \mapsto \bigvee J): \mathfrak{R}(L, \mathcal{A}) \rightarrow(L, \mathcal{A})
$$

are uniform and constitute the coreflection maps from the category of compact uniform frames to that of uniform frames, called the Samuel compactification (see [4]).

Note. Compare this with the Stone-Čech compactification as constructed in [3]. There, one considers the completely regular ideals $J$ in the sense that for $a \in J$ there is a $b \in J$ such that $a \prec b$. Since $\prec$ is just $\triangleleft_{\mathfrak{F} L}$, this means that the Stone-Čech compactification is the Samuel compactification of $(L, \mathfrak{F} L)$.

1.7. Recall that a frame homomorphism $h$ is dense if $h(a)=0$ implies $a=0$. For nearness frames $(L, \mathcal{A})$ and $(M, \mathcal{B})$, a strict surjection $h:(L, \mathcal{A}) \rightarrow(M, \mathcal{B})$ (dense surjection in [5])) is a dense onto uniform homomorphism such that

(1) $\mathcal{B}=\{h[A] \mid A \in \mathcal{A}\}$, and

(2) $\left\{h_{*}[B] \mid B \in \mathcal{B}\right\}$, where $h_{*}$ is the right Galois adjoint of $h$, is a basis of $\mathcal{A}$.

(Note that $(2)$ is automatic in case of uniform frames.)

A nearness frame $(L, \mathcal{A})$ is complete if every strict surjection $(M, \mathcal{B}) \rightarrow$ $(L, \mathcal{A})$ is an isomorphism. A completion of $(L, \mathcal{A})$ is a strict surjection $\gamma: \mathbf{C}(L, \mathcal{A}) \rightarrow(L, \mathcal{A})$ where $\mathbf{C}(L, \mathcal{A})$ is complete. It exists and is unique up to isomorphism $([5,9,12,14])$. 
1.7.1. The completion of a uniform frame $(L, \mathcal{A})$ can be constructed via the Samuel compactification (see [4]). One takes $\mathfrak{R}(L, \mathcal{A})$ and considers

$$
\mathrm{C}(L, \mathcal{A})=\mathfrak{R}(L, \mathcal{A}) / R
$$

where $R$ is the congruence generated by

$$
\{(\bigvee\{\mathfrak{k}(a) \mid a \in A\}, L) \mid A \in \mathcal{A}\} \quad \text { where } \quad \mathfrak{k}(a)=\left\{x \mid x \triangleleft_{\mathcal{A}} a\right\}
$$

(that is, one factorizes so as to make the $\{\mathfrak{k}(a) \mid a \in A\}$ covers).

1.7.2. If $(L, \mathcal{A})$ is a general nearness frame one can construct a completion $\mathbf{C}(L, \mathcal{A})$ as $\mathfrak{D} L / R$ where $\mathfrak{D} L$ is the down-set frame and $R$ is the congruence on $L$ generated by

$$
\{\mathfrak{k}(a), \downarrow a) \mid a \in L\} \cup\{(\bigvee\{\downarrow a \mid a \in A\}, L) \mid A \in \mathcal{A}\}
$$

endowed with the nearness $\mathcal{A}^{\downarrow}$ generated by $\{\{\downarrow a \mid a \in A\} \mid A \in \mathcal{A}\}$. This leads to $\mathbf{C}(L, \mathcal{A})$ consisting of the down-sets $U$ satisfying

(R1) $\mathfrak{k}(a) \subseteq U \Rightarrow a \in U$, and

(R2) $\exists C \in \mathcal{A}$ such that $\{a\} \wedge C \subseteq U \Rightarrow a \in U$.

More precisely, the completion is the strict surjection

$$
\gamma_{(L, \mathcal{A})}: \mathbf{C}(L, \mathcal{A}) \rightarrow(L, \mathcal{A})
$$

where $\gamma_{(L \cdot \mathcal{A})}(U)=\bigvee U$, with the right Galois adjoint given by

$$
\gamma_{*}(a)=\downarrow a .
$$

\section{Near COmpactness and Samuel NeAR-COMPaCtification}

2.1. A regular frame will be called near-compact if it is complete with respect to some totally bounded nearness. To motivate this terminology recall that for completely regular frames $L$,

$L$ is compact iff it is complete with respect to some totally bounded uniformity.

Thus we have a natural concept parallel to compactness suitable for general regular frames. Note, however, that even a completely regular frame can be near-compact without being compact - see 2.6 below; on the other hand, if a regular frame is compact then it is actually completely regular.

In a different direction one has from [1] that

(2.1.1) the completion of a nearness frame is compact iff the nearness is a totally bounded uniformity

and 
(2.1.2) a complete nearness frame is compact iff it is totally bounded uniform.

2.2. One cannot expect a very nice behaviour of the class of nearcompact frames, mainly because the completion of general nearness frames is not functorial ([2], see 3.3 below), a nearness larger than a complete one is not necessarily complete, and other anomalies of the non-uniform nearnesses. However, independently of functoriality, completion of nearness frames generally preserves coproducts $([15])$. Consequently, we have the counterpart of the point-free Tychonoff theorem any coproduct of near-compact frames is near-compact.

2.3. Extending the concept of surjection ([5]) we call an onto uniform homomorphism $h:(M, \mathcal{B}) \rightarrow(L, \mathcal{A})$ between two nearness frames with $\mathcal{B}$ totally bounded a tb-surjection if the image of $\mathcal{B}$ under $h$ is $\operatorname{tb} \mathcal{A}$. In this sense we speak of dense tb-surjection and strict tb-surjection (recall $1.7)$.

Define

$$
\mathfrak{S}(L, \mathcal{A})=\mathbf{C}(L, \mathrm{tb} \mathcal{A})
$$

Hence we have the strict tb-surjections

$$
\sigma_{(L, \mathcal{A})}: \mathfrak{S}(L, \mathcal{A}) \rightarrow(L, \mathcal{A})
$$

composing the completion maps $\gamma_{(L, \mathrm{tb} \mathcal{A})}$ with the identical embeddings $(L, \operatorname{tb} \mathcal{A}) \rightarrow(L, \mathcal{A})$.

2.3.1. Proposition. Up to isomorphism, $\sigma_{(L, \mathcal{A})}: \mathfrak{S}(L, \mathcal{A}) \rightarrow(L, \mathcal{A})$ is the unique strict tb-surjection $h:(M, \mathcal{B}) \rightarrow(L, \mathcal{A})$ with $(M, \mathcal{B})$ complete.

Proof. $(M, \mathcal{B})$ is complete and $h$ decomposes as

$$
(M, \mathcal{B}) \stackrel{k}{\longrightarrow}(L, \mathrm{tb} \mathcal{A}) \stackrel{\mathrm{id}_{L}}{\longrightarrow}(L, \mathcal{A}) .
$$

Now se the unicity of completion for $k$.

2.3.2. Note that $\mathfrak{S}(L, \mathcal{A})$ is compact uniform whenever $(L, \mathcal{A})$ is uniform (see (2.1.1)) so that we have obtained an alternative description of the Samuel compactification of uniform frames ([4], 1.7.1).

Consequently we will refer to $\sigma_{(L, \mathcal{A})}: \mathfrak{S}(L, \mathcal{A}) \rightarrow(L, \mathcal{A})$ in the general case as the Samuel near-compactification of $(L, \mathcal{A})$.

\subsection{An alternative description of Stone-Čech compactifica-} tion. Recall the Stone-Čech compactification from [3]. There, for a 
completely regular frame $L$, one took the frame $\mathfrak{K} L$ of completely regular ideals on $L$; it turns out that the map $v_{L}=(J \mapsto \bigvee J): \mathfrak{K} L \rightarrow L$ is a dense onto frame homomorphism, with right adjoint

$$
\mathfrak{k}_{L}=(a \mapsto\{x \mid x \prec a\}): L \rightarrow \mathfrak{K} L,
$$

and constitutes the compact coreflection map.

Now consider for a completely regular $L$ the fine uniformity $\mathfrak{F} L$ and its totally bounded part $\operatorname{tb} \mathfrak{F} L$. Then $\mathbf{C}(L, \operatorname{tb} \mathfrak{F} L)$ is compact by (2.1.2) and (1.5.1), and applying the underlying frame functor $|\cdot|$ to $\left.\gamma_{(L, \mathrm{tb} F} L\right): \mathbf{C}(L, \operatorname{tb} \mathfrak{F} L) \rightarrow(L, \operatorname{tb} \mathfrak{F} L)$ we obtain $\gamma_{L}:|\mathbf{C}(L, \operatorname{tb} \mathfrak{F} L)| \rightarrow L$, a homomorphism to $L$ from a compact completely regular frame. Further, for any other such $h: M \rightarrow L$ we have the following commutative square

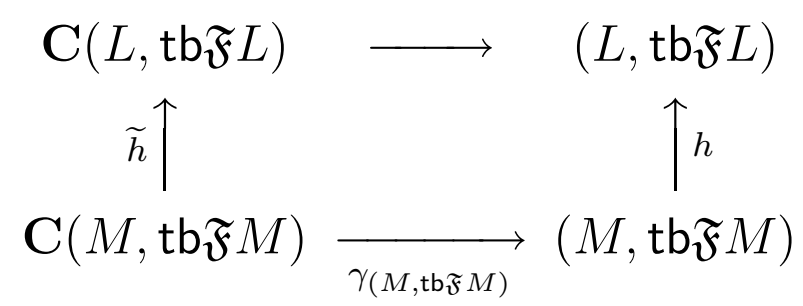

where the bottom map is an isomorphism by compacthess. Hence, acting the functor $|\cdot|$ produces the factorization $h=\gamma_{L} \bar{h}$, where $\bar{h}$ is unique since $\gamma_{L}$ is dense, showing that $\gamma_{L}$ is the coreflection map from compact completely regular frames.

2.5. For any regular frame $L$, its fine near-compactification will be the underlying frame homomorphism of the completion map

$$
\gamma_{(L, \mathrm{tbc} L)}: \mathbf{C}(L, \operatorname{tb} \mathfrak{C} L) \rightarrow(L, \operatorname{tb} \mathfrak{C} L),
$$

denoted by $\delta_{L}: \mathfrak{N} L \rightarrow L$. Note this is characterized as a dense frame homomorphism $h: M \rightarrow L$ for which $\left\{h_{*}[A] \mid A \in \operatorname{tb} \mathfrak{C} L\right\}$ generates a complete nearness on $M$

2.5.1. Remark. It should be emphasized that the Stone-Čech compactification of a completely regular $L$ is not necessarily its fine near-compactification. Take a completely regular $L$ that is not normal, and $v=v_{L}: \mathfrak{K} L \rightarrow L$. Because of the standard fact that $v[A] v[B] \leq$ $v[A B]$ each cover of $L$ of the form $v[A]$, with $A$ a cover of $\mathfrak{K} L$, has a star-refinement. Since $L$ is not normal there exists a finite cover $B$ of $L$ that has no star-refinement. Thus, $v_{*}[B]$ cannot be a cover because $v\left[v_{*}[B]\right]=B$.

2.6. Proposition. For any regular $L$, if its fine near-compactification is normal then $L$ it is normal. 
Proof. If $a \vee b=1$ in $L$ then $\{a, b\}$ is a uniform cover of $(L$, tbc $)$, so $\{\downarrow a, \downarrow b\}$ is a cover of $\mathfrak{N} L$, hence there exist $U, V \in \mathfrak{N} L$ such that $U \cap V=\downarrow 0$ and $(\downarrow a) \vee U=\downarrow 1=\downarrow b \vee V$. Then we have for the completion map $\gamma: \mathfrak{N} L=\mathbf{C}(L, \operatorname{tbc} L) \rightarrow(L$, tbc $L)$ that $a \vee \gamma(U)=1=b \vee \gamma(V)$, $\gamma(U) \wedge \gamma(V)=0$, showing $L$ is normal.

Corollary. For a regular $L$ that is not normal, $\mathfrak{N} L$ is not compact. Hence, it is near-compact but not compact.

2.7. Proposition. A near-compact regular frame is compact iff it is normal.

Proof. $\Rightarrow$ : Any compact regular frame is normal,

$\Leftarrow$ : Regular and normal implies that $\prec$ interpolates, hence the finite cover nearness is a uniformity. Thus $L$ is complete in a totally bounded uniformity, and therefore compact.

\section{More on $\mathfrak{N} L$, And COMPARIson of two Completions}

3.1. The Stone-Čech compactification $\mathfrak{K} L$ in [3] (see 2.4) was obtained as a subframe of the ideal frame $\mathfrak{J} L$. In fact, the $\mathfrak{N} L$ is also a frame of particular ideals, although specified differently. We have

Proposition $\mathfrak{N} L$ isisomorphic to the frame of the ideals $J \subseteq L$ such that

$$
\{x \mid x \prec a\} \subseteq J \quad \Rightarrow \quad a \in L .
$$

Proof. We will prove that the down-sets $U \subseteq L$ describing $\mathbf{C}(L, \mathrm{tb} L)$ are precisely the mentioned ideals.

First note that $\triangleleft_{\mathrm{tbC} L}=\prec$ (for $a \prec b$ consider the cover $\left\{a^{*}, b\right\}$ and the fact that $\left.\left\{a^{*}, b\right\} a \leq b\right)$ so that $\{x \mid x \prec a\}$ is the corresponding $\mathfrak{k}(a)$. Now let $U$ satisfy (R1) and (R2). Then we have $(*)(\equiv(\mathrm{R} 1))$; secondly, if $a, b \in U$ and $x \prec a \vee b$ then $\left\{x^{*}, a, b\right\}$ is a finite cover and $x \wedge x^{*}, x \wedge a, x \wedge b \in U$ so that $x \in U$. Using (*) again we obtain $a \vee b \in U$.

On the other hand, if $U$ is an ideal satisfying $(*)$ then it is a downset satisfying (R1); further, if $C=\left\{c_{1}, \ldots, c_{n}\right\}$ is a finite cover and $\{a\} \wedge$ $C \subseteq U$ then $a=\bigvee\{a\} \wedge C \in U$ since $U$ is an ideal $a \wedge c_{i} \in U$ and since it is a ideal,

3.1.1. In particular, for a Boolean frame $L$,

$$
\mathfrak{N} L=\mathfrak{J} L=\mathfrak{K} L
$$

as $a \prec a$ here. 
3.2. For any completely regular frame $L$, the general facts about strong nearness frames and completions supply a uniform homomorphism $h: \mathfrak{K} L \rightarrow \mathfrak{N} L$ such that $h v_{*}=\left(\nu_{*}\right)^{\circ}$ for the completion maps

$$
v: \mathfrak{K} L \rightarrow L \quad \text { and } \quad \nu: \mathfrak{N} L \rightarrow L
$$

where $v_{*}(a)=\mathfrak{k}(a), \nu_{*}(a)=\downarrow a$, and

$$
\left(\nu_{*}\right)^{\circ}(a)=\bigvee\left\{\nu_{*}(x) \mid x \prec a\right\}=\bigvee\{\downarrow x \mid x \prec a\}=\mathrm{n}(\mathfrak{k}(a)),
$$

(notation from $[2], 2) \mathrm{n}$ being the nucleus (1.1) in $\mathfrak{J} L$ which determines $\mathfrak{N} L$.

Proposition. For any $L, h$ is a frame embedding and it is an isomorphism iff $L$ is normal.

Proof. For any $J \in \mathfrak{J} L$,

$$
\begin{gathered}
h(J)=\bigvee\{h(\mathfrak{k}(a)) \mid a \in J\}=\bigvee\left\{h v_{*}(a) \mid a \in J\right\}= \\
=\bigvee\{\mathfrak{k}(a) \mid a \in J\}=\mathrm{n}(J) .
\end{gathered}
$$

Thus, $J=\downarrow 0$ whenever $h(J)=\downarrow 0$, that is, $h$ is dense, and hence one-one by compactness and regularity.

Further, if $h$ is an isomorphism then $\mathfrak{N} L$ is compact and by [1] this makes the finite cover nearness of $L$ a uniformity which in turn implies $L$ is normal. Conversely, for normal $L, \mathfrak{N} L$ is a Stone-Čech compactification of $L$ and hence $h$ is an isomorphism.

Note. In particular, for compact (completely) regular $L, \mathfrak{N} L \rightarrow L$ is an isomorphism iff $L$ is normal.

3.3. The Stone-Cech compactification is functorial, indeed the coreflection from the subcategory of compact regular frames. The same holds for the Samuel compactification of uniform frames. The question naturally arises as to the functoriality of the near-compactification $\mathfrak{N} L \rightarrow L$. The ansver is negative; the result 3.4 in [2] translates in our terminology to

Proposition. The correspondence $L \mapsto \mathfrak{N} L$ is not functorial with $\gamma_{L}: \mathfrak{N} L \rightarrow L$ natural.

Proof. We give an alternative proof which fits better into the present context.

Let $N$ be any regular frame which is extremally disconnected, that is, with $a^{*} \vee a^{* *}=1$ for all $a$. As is well known, this makes the Boolean frame

$$
\mathfrak{B N}=\left\{a \in N \mid a=a^{* *}\right\}
$$


a bounded sublattice of $N$ so that the map

$$
\mathfrak{B} N \stackrel{\varepsilon=(b \mapsto b)}{\longrightarrow} N \stackrel{\left(\gamma_{L}\right)_{*}=(b \mapsto \downarrow b)}{\longrightarrow} \mathfrak{N} N
$$

is a bounded lattice homomorphism (note that by $3.1, \downarrow(b \vee c)=\downarrow b \vee \downarrow c$ in $\mathfrak{N} N)$; further, such a lattice homomorphism is known to determine a frame homomorphism $k: \mathfrak{J}(\mathfrak{B} N) \rightarrow \mathfrak{N} N$ such that $k(\downarrow b)=\downarrow b$ for all $b \in \mathfrak{B} N$. Now $\mathfrak{N} M=\mathfrak{J} M$ as noted in 3.1.1, and supposing $L \mapsto \mathfrak{N} L$ is functorial as required we have the following commuting diagram

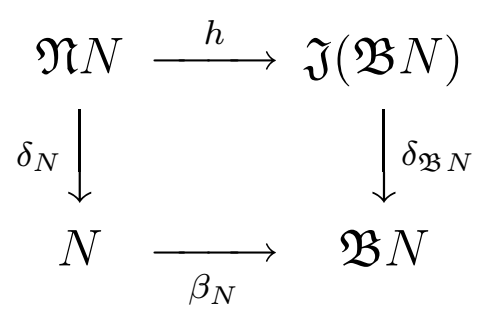

with $\beta_{N}(a)=a^{* *}$ and $\delta_{\mathfrak{B} N}(J)=\bigvee J$ (in $\left.\mathfrak{B} N\right)$. Thus $\delta_{\mathfrak{B} N} h=\beta_{N} \delta_{N}$ as well as $k\left(\delta_{\mathfrak{B} N}\right)_{*}=\left(\delta_{N}\right)_{*} \varepsilon$ and combining these we obtain

$$
\delta_{\mathfrak{B} N}\left(\delta_{\mathfrak{B} N}\right)_{*}=\operatorname{id}_{\mathfrak{B} N}=\beta_{N} \varepsilon=\beta_{N} \delta_{N}\left(\delta_{N}\right)_{*} \varepsilon=\delta_{\mathfrak{B} N} h k\left(\delta_{\mathfrak{B} N}\right)_{*}
$$

so that $\delta_{\mathfrak{B} N}=\delta_{\mathfrak{B} N} h k$ because $\left(\delta_{\mathfrak{B} N}\right)_{*}[\mathfrak{B} N]$ generates $\mathfrak{J}(\mathfrak{B} N)$. Consequently, $h k=$ id since $\delta_{\mathfrak{B} N}$ is dense and therefore monic, and as $h$ is also dense ( $\beta_{N}$ being dense) this makes it an isomorphism; hence $\mathfrak{N} N$ is compact and by 2.7 this makes $N$ normal. However, there do exist extremally disconnected regular frames which are not normal, such as the frame of open sets of the Gleason cover $X$ of the Tychonoff plank $[7,6])$.

3.4. Samuel near-compactifications and completions. We begin with a general result, to be used as a crucial tool later on.

3.4.1. Lemma. For any nearnesses $\mathcal{A}$ and $\mathcal{B}$ on a regular frame $L$ such that $\mathcal{B} \subseteq \mathcal{A}$ and $\triangleleft_{\mathcal{B}}=\triangleleft_{\mathcal{A}}$ there exists a unform frame homomorphism $h: \mathbf{C}(L, \mathcal{B}) \rightarrow \mathbf{C}(L, \mathcal{A})$ such that $h(\downarrow a)=\downarrow a$ and $h_{*}(\downarrow a)=\downarrow a$.

Proof. For the nuclei $l_{\mathcal{A}}$ and $l_{\mathcal{B}}$ on $\mathfrak{D} L$ that determine $\mathbf{C}(L, \mathcal{A})$ resp. $\mathbf{C}(L, \mathcal{B})$ the given condition imply that $l_{\mathcal{B}} \leq l_{\mathcal{A}}$; hence $\mathbf{C}(L, \mathcal{A})$ is the image of $\mathbf{C}(L, \mathcal{B})$ under the action of $l_{\mathcal{A}}$ and we can define $h$ as the map induced by this, Then, obviously, $h(\downarrow a)=\downarrow(a)$ for all $a \in L$ and by the definition of the nearness involved it is then clear that $h$ is a uniform frame homomorphism. Further, $h(\downarrow a)=\downarrow(a)$ means that $\gamma_{(L, \mathcal{A})} h=\varepsilon \gamma_{(L, \mathcal{B})}$ for the completion maps for $(L, \mathcal{A})$ and $(L, \mathcal{B})$ and the uniform homomorphism $\varepsilon:(L, \mathcal{B}) \rightarrow(L, \mathcal{A})$ mapping $L$ identically. Consequently $h_{*}\left(\gamma_{(L, \mathcal{A})}\right)_{*}=\left(\gamma_{(L, \mathcal{B})}\right)_{*} \varepsilon_{*}$ which says that $h_{*}(\downarrow a)=\downarrow a$ for all $a \in L$, as claimed. 
3.4.2. For any nearness frame $(L, \mathcal{A})$, recall from 2.3 .2 that its Samuel near-compactification is

$$
\sigma_{(L, \mathcal{A})}=\mathbf{C}(L, \mathrm{tb} \mathcal{A}) \stackrel{\gamma_{(L, \mathrm{tb} \mathcal{A})}}{\longrightarrow}(L, \mathrm{tb} \mathcal{A}) \stackrel{\mathrm{id}_{L}}{\longrightarrow}(L, \mathcal{A}) .
$$

Note that for uniform $(L, \mathcal{A})$, this is its Samuel compactification as introduced in [4]. On the other hand, the Samuel near-compactification of any nearness frame determines its completion as follows.

3.4.3 Proposition. For any nearness frame $(L, \mathcal{A})$, if

$\mathrm{C}(L, \mathrm{tb} \mathcal{A})=(M, \mathcal{B})$ $\sigma=\sigma_{(L, \mathcal{A})}:(M, \mathcal{B}) \rightarrow(L, \mathcal{A})$

$\widetilde{M}=M / \Theta$ for the congruence $\Theta$ on $M$ generated by the pairs $\left(\bigvee \sigma_{*}[A], 1\right), A \in \mathcal{A}$,

$\nu: M \rightarrow \widetilde{M}$ is the corresponding quotient homomorphism, $\sigma=\tilde{\sigma} \nu$, and

$\widetilde{\mathcal{A}}$ is the nearness on $\widetilde{M}$ generated by $\left\{\nu \sigma_{*}[A] \mid A \in \mathcal{A}\right\}$,

then $\widetilde{\sigma}:(\widetilde{M}, \widetilde{\mathcal{A}}) \rightarrow(L, \mathcal{A})$ is the completion of $(L, \mathcal{A})$.

Proof. To begin with, it is obvious that $\sigma$ factors as stated and the $\nu \sigma_{*}[A], A \in \mathcal{A}$ are covers of $\widetilde{M}: \sigma\left(\bigvee \sigma_{*}[A]\right)=\bigvee \sigma \sigma_{*}[A]=\bigvee A=1$ and $\nu\left(\bigvee \sigma_{*}[A]\right)=1$ by the definition of $\nu$. Further, $\widetilde{\mathcal{A}}$ is a nearness on $\widetilde{M}$ : if $x \triangleleft_{\mathcal{B}} a$ in $M$ so that $B x \leq a$ for some $B \in \mathcal{B}$ then, by the properties of $\sigma$, we have $\sigma_{*}[C] \leq B$ for some $C \in \operatorname{tb} \mathcal{A}$, hence also $\sigma_{*}[C] x \leq a$ which implies $\nu \sigma_{*}[C] \nu(x) \leq \nu(a)$, therefore $\nu(x) \triangleleft_{\widetilde{A}} \nu(a)$, and finally $\nu(a)=\bigvee\left\{\nu(x) \mid \nu(x) \triangleleft_{\widetilde{\mathcal{A}}} \nu(a)\right\}$ by the properties of $\mathcal{B}$.

Next, $\widetilde{\sigma}$ is a strict surjection: it is obviously dense onto, and strict because $\tilde{\sigma}\left(\nu \sigma_{*}[A]\right)=A$ and

$$
\nu \sigma_{*}[A]=\nu \nu_{*}(\widetilde{\sigma})_{*}[A]=(\widetilde{\sigma})_{*}[A]
$$

for each $A \in \mathcal{A}$. Now it follows from [1] 3.4 that there exists a strict surjection $g: \mathbf{C}(L, \mathcal{A}) \rightarrow(\widetilde{M}, \widetilde{\mathcal{A}})$ such that $\widetilde{\sigma} g=\gamma_{(L, \mathcal{A})}$. On the other hand, we have $h:(M, \mathcal{B}) \rightarrow \mathbf{C}(L, \mathcal{A})$ with $\gamma_{(L, \mathcal{A})} h=\sigma$ by the lemma, because $\triangleleft_{\mathrm{tb} \mathcal{A}}=\triangleleft_{\mathcal{A}}$. Now $\sigma_{*}=h_{*}\left(\gamma_{(L, \mathcal{A})}\right)_{*}$ so that $h \sigma_{*}=\left(\gamma_{(L, \mathcal{A})}\right)_{*}$, and hence, for any $A \in \mathcal{A}$,

$$
h\left(\bigvee \sigma_{*}[A]\right)=\bigvee h \sigma_{*}[A]=\bigvee\left(\gamma_{(L, \mathcal{A})}\right)_{*}[A]=1,
$$

the last step by the properties of completions. Next, by the definition of $(\widetilde{M}, \widetilde{\mathcal{A}})$, there exists $l:(\widetilde{M}, \widetilde{\mathcal{A}}) \rightarrow \mathbf{C}(L, \mathcal{A})$ such that $l \nu=h$. As a result,

$$
\widetilde{\sigma} g l \nu=\widetilde{\sigma} g h=\gamma_{(L, \mathcal{A})} h=\sigma=\widetilde{\sigma} \nu
$$


and hence $g l=$ id because $\widetilde{\sigma}$ is dense and $\nu$ isonto. Finally, since $g$ is dense this makes it an isomorphism, showing $\widetilde{\sigma}$ is the completion of $(L, \mathcal{A})$.

3.4.4. Remarks. 1. It is an open question whether the proposition can be proved without reference to the existence of the completion of $(L, \mathcal{A})$; of course this was done in the uniform case $([4])$, based on the properties of compact uniform frames, but whether this is still possible in the general case is not clear.

2. There is a natural generalization of the initial part of the proposition as follows. Calling a uniform homomorphism $h:(M, \mathcal{B}) \rightarrow(L, \mathcal{A})$ relatively strict if $\mathcal{B}$ is generated by the $h_{*} h[B], B \in \mathcal{B}$, one readily obtains the following by the same arguments used in the above proof: Any dense onto relatively strict homomorphism $h:(M, \mathcal{B}) \rightarrow(L, \mathcal{A})$ has a factorization

$$
(M, \mathcal{B}) \stackrel{\nu}{\longrightarrow}(\widetilde{M}, \widetilde{\mathcal{A}}) \stackrel{\widetilde{h}}{\longrightarrow}(L, \mathcal{A}),
$$

with the same $\widetilde{M}, \nu, \widetilde{h}, \widetilde{\mathcal{A}}$ as before, where $\widetilde{h}$ is a strict surjection. This then applies to any Samuel near-compactification $\sigma:(M, \mathcal{B}) \rightarrow(L, \mathcal{A})$ so that one obtains the strict surjection $\widetilde{\sigma}:(\widetilde{M}, \widetilde{\mathcal{A}}) \rightarrow(L, \mathcal{A})$ and then also the required $g: \mathbf{C}(L, \mathcal{A}) \rightarrow(\widetilde{M}, \widetilde{\mathcal{A}})$ with $\widetilde{\sigma} g=\gamma_{\mathcal{A}}$. Only the remaining step to show $g$ is an isomorphism requires the particular properties of $\operatorname{tb} \mathcal{A} \subseteq \mathcal{A}$ which make the original lemma applicable.

\section{REFERENCES}

[1] B. Banaschewski, Uniform Completion in Pointfree Topology; in: Top. and Alg. Structures in Fuzzy Sets (eds. S.E. Rodabaugh and E.P. Klement), Kluwer 2003. 19-56.

[2] B. Banaschewski, S.S. Hong and A. Pultr, On the completion of nearness frames, Quaestiones Mathematicae 21 (1998), 19-37

[3] B. Banaschewski and C.J. Mulvey, Stone - Čech compactification of locales, Houston J. Math. 6 (1980), 301-312.

[4] B. Banaschewski and A. Pultr, Samuel compactification and completion of uniform frames, Math. Proc. Cambridge Phil. Soc. 108 (1990), 63-78.

[5] B. Banaschewski and A. Pultr, Cauchy points of uniform and nearness frames, Quaestiones Math. 19(1-2) (1996), 101-127.

[6] E.K. van Dowen, Compactness-like properties and nonnormality of the space of nonstationary ultrafilters, Topology and Applications 51 (1993), 73-79.

[7] A.M. Gleason, Projective Topological Spaces, Illinois J. Math., 2, Issue 4A (1958), 482-489.

[8] H. Herrlich, A concept of nearness, Gen. Top. and its Appl. 4 (1974), 191-212.

[9] J.R. Isbell, Atomless parts of spaces, Math. Scand. 31 (1972), 5-32.

[10] J.R. Isbell, Uniform Spaces, Mathematical Surveys Nr. 12, American Mathematical Society (1964). 
[11] P.T. Johnstone, Stone Spaces, Cambridge Sudies in Advanced Math. no 3, Cambridge University Press, 1983.

[12] I. Kříž, $A$ direct description of uniform completion in locales and a characterization of LTgroups, Cahiers de Top. et Géom. Diff. Cat. XXVII (1986), 19-34.

[13] S. Mac Lane, Categories for the Working Mathematician, Springer-Verlag, New York, 1971.

[14] A. Pultr, Frames, Chapter in: Handbook of Algebra, Vol.3, (Ed. by M. Hazewinkel), Elsevier 2003, 791-858.

[15] A. Pultr and A. Tozzi, Completion and coproduct of neraness frames, Proc. Symposium on Categorical Topology, University of Cape Town 1994, 177-186.

Department of Mathematics and Statistics, McMaster University, 1280 Main St. W, Hamilton, Ontario L8S 4K1, Canada

Department of Applied Mathematics and ITI, MFF, Charles UniverSity, CZ 11800 Praha 1, Malostranské nám. 25, Czech Republic

E-mail address: pultr@kam.ms.mff.cuni.cz 